\title{
Cloud Web-Service Model for Detection of Lineaments and Circular Structures in Space Images
}

\author{
Aleksey A. Buchnev, Pavel A. Kim, \\ Valeriy P. Pyatkin, Gennadiy I. Salov* \\ Institute of Computational Mathematics \\ and Mathematical Geophysics SB RAS \\ 6 Akademika Lavrentieva, Novosibirsk, 630090, Russia
}

We consider cloud Web-service model to detect abnormal (linear and circular) structures on the space images, which solves the problem of providing new services to Earth remote sensing data processing within the cloud SaaS model. Service model implemented on the Windows platform.

Keywords: remote sensing, cloud computing, lineaments, circular structures.

\section{Макет облачного web-сервиса}

\section{по выделению линеаментов и кольцевых структур на космических изображениях}

\author{
А.А. Бучнев, П.А. Ким, В.П. Пяткин, Г.И. Салов \\ Институт вычислительной математики \\ и математической геофизики СО РАН \\ Россия, 630090, Новосибирск, пр. Академика Лаврентьева, 6
}

Рассматривается макет Web-сервиса для выделения аномальных (линейных и кольиевых) структур на космических изображениях, который решает новую задачу предоставления услуг обработки данных дистанционного зондирования Земли в рамках облачной модели SaаS. Макет сервиса реализован на платформе Windows.

Ключевые слова: дистанциионное зондирование, облачные вычисления, линеаменты, кольцевые структуры.

(C) Siberian Federal University. All rights reserved

* Corresponding author E-mail address: baa@ooi.sscc.ru,pvp@ooi.sscc.ru 


\section{Введение}

Программа прикладных дистанционных исследований Земли из космоса обширна и разнообразна [1]. Эффективность этих исследований во многом определяется используемыми методами тематической обработки данных дистанционного зондирования Земли (ДЗ3). Функциональные возможности разработанного в ИВМиМГ СО РАН совместно с ФГБУ «НИЦ «Планета» Росгидромета РФ программного комплекса PlanetaMonitoring для обработки данных ДЗ3 дают возможность использовать при их обработке практически функционально полный набор операций [2]. Одна из важных операций, реализованных в комплексе, - обнаружение объектов заданной формы (линеаментов и кольцевых структур) на космических снимках (программа LINECOIL). Материалы аэрокосмических съемок показали повсеместное распространение линейных и кольцевых образований в структуре земной коры, что обусловило широкий интерес к ним со стороны исследователей, изучающих нашу планету.

При обработке космических снимков с целью обнаружения на них объектов, представляющих интерес, в силу целого ряда причин предпочтение отдается статистическому подходу [3-5]. Основная причина этого в том, что вследствие случайного характера природных процессов данные дистанционных измерений (спектросовмещенные изображения) содержат много случайных вариаций, маскирующих различия значений яркости изображения в точках области объекта и в точках области фона. В подобной ситуации надежные алгоритмы обнаружения могут быть построены только с помощью вероятностного (статистического) подхода.

Есть целый ряд задач Д33, для которых очень важна задача выделения линейных и кольцевых структур на космических изображениях. Прежде всего это геологические исследования Земли из космоса. Так, обработка аэрокосмических изображений (АИ) восточной части Сибирской платформы Якутской кимберлитоносной провинции показала геологически значимую корреляцию выделенной статистическим методом системы линейных элементов и типов зон разрывных нарушений. Статистический метод выделения линейных и кольцевых структур на АИ оказался достаточно эффективным при решении ряда задач экологических исследований Земли из космоса. В проблеме космической охраны Земли существует настоятельная необходимость изучения процессов падения небесных тел на Землю и картирование мест их падения. Два исторических космических факта подчеркивают актуальность решения проблемы выделения импактных кратеров на космических снимках. Это, безусловно, падение на Землю Тунгусского метеорита (1908 г.) и столкновение кометы Шумейкера-Леви с планетой Юпитер (1994 г.).

Еще одна задача экологической программы исследования Земли из космоса связана с изучением и картированием сейсмоопасных регионов по данным цифровой обработки АИ и комплекса наземных наблюдений. С использованием статистического подхода была проведена автоматизированная обработка космических снимков района Спитакского землетрясения (1988 г.) и выделены две новые крупные дизъюнктивные зоны, не совпадающие с зонами известных глубинных разломов и, по-видимому, играющие более существенную роль в тектонической структуре территории, чем это представлялось ранее. В последнее время алгоритм линеаментного анализа АИ используется в мониторинге ледовой обстановки в полярных районах Земли в практике оперативной работы ФГБУ «НИЦ «Планета» Росгидромета РФ.

$$
-742-
$$


В настоящее время становится актуальным использование Internet-технологий для оперативной интеграции информационно-вычислительных ресурсов для решения задач обработки данных Д33. Новая парадигма облачных вычислений (cloud computing) даёт такую возможность [6]. Основная идея облачных вычислений - технологии распределённой обработки и хранения данных, в которых все необходимые ресурсы предоставляются пользователю как Internetсервис. Концептуально технологию облачных вычислений делят на предоставление инфраструктуры в качестве сервиса (IaaS, Infrastructure as a Service), платформы в качестве сервиса (PaaS, Platform as a Service) или программного обеспечения в виде сервиса (SaaS, Software as a Service), а также многих придуманных и ещё не придуманных Internet-технологий для удалённых пользовательских вычислений. В данной работе основное внимание будет уделено облачной концепции SaaS. Рассмотрим разработку макета Web-сервиса по выделению линейных и кольцевых структур, которая решает новую задачу предоставления услуг обработки данных Д33 в рамках облачной модели SaaS. Макет сервиса реализован на платформе Windows и coстоит из следующих компонент: вычислительной компоненты, созданной на основе разработанного ранее Windows-приложения LINECOIL выделения линейных и кольцевых структур на спутниковых изображениях, и Web-интерфейса, выполненного на основе свободного Webсервера Apache.

\section{Web-интерфейс}

Текущая реализация Web-интерфейса продолжает многолетнее развитие разрабатываемой в ИВММГ СО РАН концепции объединения в рамках дной программной оболочки разнородных алгоритмов для обработки изображений. Таким образом, разные вычислительные компоненты опираются на единообразный сетевой интерфейс, предусматривающий возможность реализации таковых компонент на различных архитектурных платформах гетерогенной сети, включая многопроцессорные архитектуры Сибирского суперкомпьютерного центра. Обозначенная идеология успешно укладывается в облачный подход к развитию программного обеспечения, ориентируя на отказ от прямой адресации элементов сети в пользу гипертекстовых ссылок. С учетом разнородности и обширности рынка пользовательских браузеров, используемых в Интернет-сети, WEB-интерфейс в макетном варианте реализован с применением минимальных средств гипертекстового языка HTML. Сервисная часть выполнена на Apache 2.2, но закладываемая в концепцию «многополярность» распараллеливания процессов не противоречит и другим платформам типа IIS Windows. Структурно в интерфейсе можно выделить следующие три части: подготовка параметров, вызов вычислителя, просмотр результата. Дальнейшая детализация приведет к более сложным структурам, образующим своего рода интерактивные технологические карты вычислительного процесса. О характере и сложности такой технологической карты можно получить представление на примере выбора обрабатываемого изображения, которое может оставаться неизвестным пользователю ни в форме представления, ни в масштабе, ни в палитре. Аналогичные трудности встанут перед пользователем и при анализе результатов расчета. Для упрощения экспериментального эксплуатационного этапа тестирования макета передача пользовательских изображений производится по анонимному входу в библиотеку сервиса по FTP-протоколу. Таким образом, пользователь имеет возможность 
вести поиск «скрытых» объектов как по архивным данным, так и по индивидуальным образцам изображений.

\section{Вычислительная компонента}

Основная часть процедур вычислительной компоненты состоит в нахождении статистик $S^{+}$ и $S^{-}$на основе анализа значений пикселов изображения вдоль нормалей к предполагаемому положению объекта [7]. Эти статистики определяются следующим образом. Для $i=1, \ldots, k$ обозначим через $\varsigma_{i}, \xi_{i 1}, \xi_{i 2}$ значения пикселов, наблюдаемых в точках проверяемого положения объекта и на линии $i$-й нормали к объекту слева и справа от него. Тогда $S^{+}=\sum_{i=1}^{k} I\left\{\varsigma_{i}>\max \left(\xi_{i 1}, \xi_{i 2}\right)\right\}$, $S^{-}=\sum_{i=1}^{k} I\left\{\zeta_{i}<\min \left(\xi_{i 1}, \xi_{i 2}\right)\right\}$, где $I\{\}-$. индикаторная функция события \{.\}, равная 1 или 0 в зависимости от того, произошло или не произошло событие \{.\}. Для каждого возможного положения решение о наличии объекта принимается по результату сравнения посчитанных значений статистик $S^{+}$и $S^{-}$с предопределенными пороговыми значениями, полученными на основе входных параметров. При этом предполагается, что количество проверяемых позиций объекта $k$ и расстояния вдоль нормалей для получения значений пикселов $\xi_{i 1}$ и $\xi_{i 2}$ таковы, что все величины могут рассматриваться как статистически независимые в совокупности, когда в поле зрения объект отсутствует. Используемый для построения статистик $S^{+}$и $S^{-}$анализ значений пикселов требует больших временных затрат при его последовательном выполнении. В связи с этим разработаны параллельные реализации алгоритмов обнаружения аномальных структур. Реализация достигается средствами OC Windows на уровне потоков: количество запускаемых потоков равно количеству логических процессоров. Каждый процесс (или поток), зная общее количество процессов и свой номер, определяет горизонтальную полосу изображения для обработки. При этом полосы у соседних процессов перекрываются, и глубина этого перекрытия зависит от размера обнаруживаемых объектов.

Вычислительная компонента облачной технологии выделения линеаментов и кольцевых структур является пакетным вариантом упомянутого выше Windows-приложения LINECOIL. На приведённых рисунках представлены скриншоты двух сеансов работы с макетом облачного сервиса по выделению линеаментов (рис. 1) и кольцевых структур (рис. 2), значение параметра Линеаменты/окружности:(0/1) $=0$ и 1 соответственно. В качестве исходного снимка используется космический снимок (с ИС3 Landsat 7) известного импактного кратера Нью-Квебек (Канада). Все необходимые для работы соответствующих процедур данные собраны в текстовом файле, имя которого служит фактическим параметром компоненты. Главными из этих данных являются: размер выделяемого объекта (длина линеамента или радиус круга в пикселях, как правило, задаётся интервал значений параметрами Минимальный размер объекта и Максимальный размер объекта); допустимая вероятность ложного обнаружения объекта (параметр Alpha); расстояние от предполагаемого положения объекта для измерения значений пикселей (параметр Приращение). Несколько слов о других параметрах, используемых в сеансе работы с макетом. Пусть L_R - текущий размер объекта. Параметр C_split - коэффициент увеличения значения $\mathbf{L} \_\mathbf{R}$ при переходе к обнаружению объектов следующего размера из заданного интервала размеров: $\mathbf{L} \_\mathbf{R}=\mathbf{L} \_\mathbf{R} * \mathbf{C} \_$split. Значение параметра $\mathbf{C} \_s t p$ определяет смещение начала линеамента/центра окружности при переходе к обнаружению следующего объекта: $\mathbf{d x}=\mathbf{d y}=$ $=\mathbf{L} \_\mathbf{R} * \mathbf{C} \_\mathbf{s t p}$. При $\mathbf{C} \_\mathbf{s t p}=\mathbf{0} \mathbf{d x}=\mathbf{d y}=\mathbf{1}$. Параметр Фон определяет фон для построения обнару- 


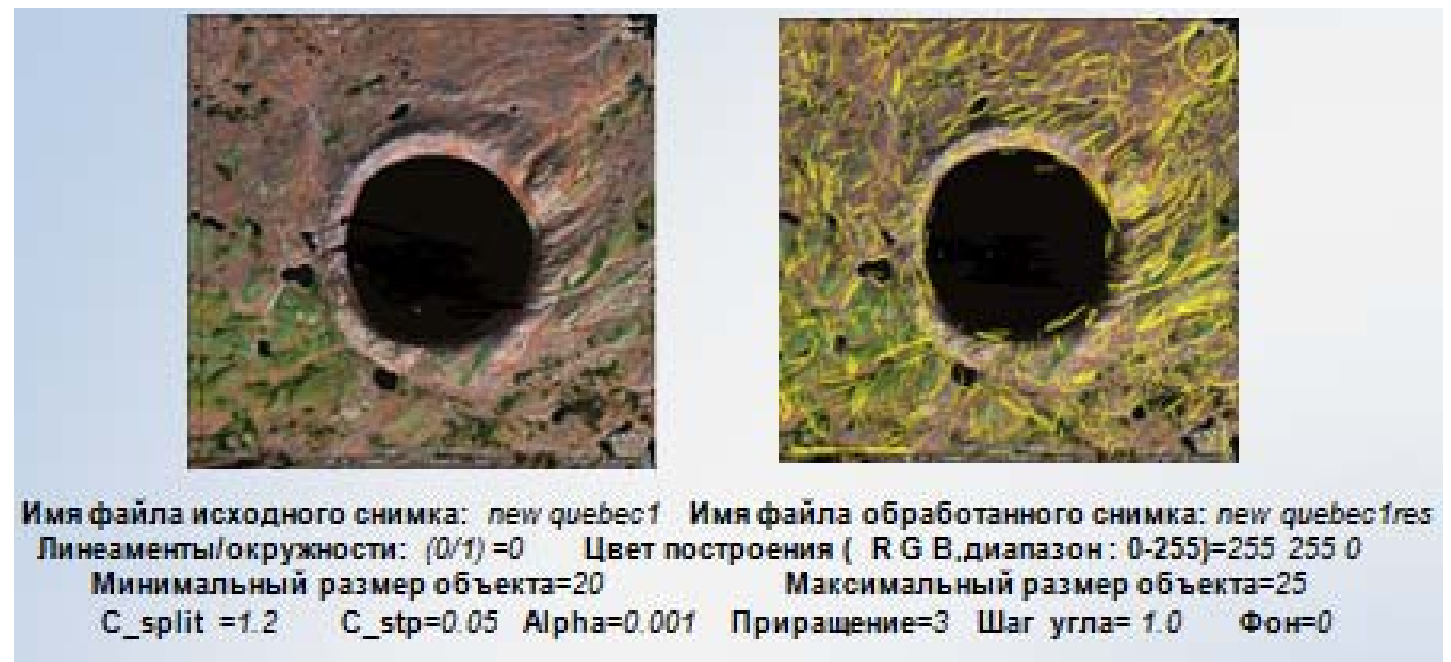

Рис. 1

Fig. 1
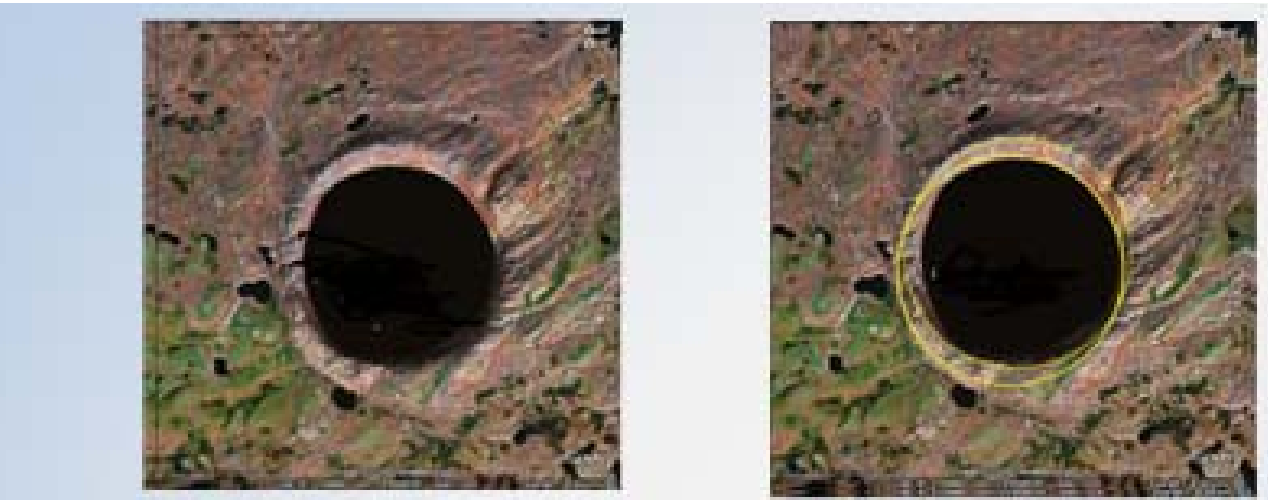

Имя файла ихходого снимка: rew quebec Имя файла обработанного снимка: new que becres ЛинеаментыКокружноти: $(0 / 1)=1$ Црет построения ( R G В диапаз сн :0-255)=255 2550 Минимальньй размер об ьекта=150 Макимальный размер об ьект а=150 C_split $=1.1 \quad$ C_stp $=0.0$ Alpha $=0.0005$ Прмрашение $=4$ шаг yтла $=1.0 \quad$ Фон $=0$

Рис. 2

Fig. 2

женных объектов: не ноль - объекты строятся на чистом поле, иначе они строятся на исходном изображении. Параметр Шаг угла задает угловое смещение (в градусах) по окружности при анализе значений пикселов. Используется только при обнаружении окружностей. Параметр Цвет построения (значения R G B, диапазон 0 - 255) задает цвет построения обнаруженных объектов. Все конкретные значения параметров (выделены курсивом в скриншотах) задаются в интерактивном режиме в данном сеансе работы с макетом. Вычислительная компонента передает в вызывающую среду код завершения, означающий либо успешное обнаружение объектов, либо ошибочный вызов (как правило, обнаруживаются неверные значения вводимых параметров). В случае успешного завершения формируются два файла с одним и тем же изображением обнаруженных объектов: один файл в формате BMP, второй - в формате JPEG. 


\section{Заключение}

Успешный опыт реализации макета облачного Web-сервиса по выделению аномальных (линейных и кольцевых) структур на космических изображениях будет использован для предоставления услуг обработки данных ДЗ3 в рамках облачной модели $\mathrm{SaaS}$ и по другим программным модулям комплекса PlanetaMonitoring, в частности блока распознавания.

Работа выполнена частично при финансовой поддержке Российского фонда фундаментальных исследований (проект № 16-07-00066) и Программы І.33П фундаментальных исследований Президиума РАН (проект № 0315-2015-0012).

\section{Список литературы}

[1] Шовенгердт Р.А. Дистанционное зондирование. Модели и методы обработки изображений. М.: Техносфера, 2010, 560 c. [Schowengerdt R.A. Remote sensing. Models and methods for image processing. Moskva, Technosfera, 2010, 560 p. (in Russian)]

[2] Асмус В.В., Бучнев А.А., Кровотынцев В.А., Пяткин В.П., Салов Г.И. Planetamonitoring: программный комплекс обработки спутниковых данных Проблемы информатики, 2013, 7(3), 85-99 [Asmus V.V., Buchnev A.A., Krovotyntsev V.A., Pyatkin V.P., Salov G.I. Planetamonitoring: software complex processing of satellite data. Problems of Informatics, 2013, 7(3), 85-99. (in Russian)]

[3] Салов Г.И. О мощности непараметрических критериев для обнаружения протяженных объектов на случайном фоне. Автометрия, 1997, 34(3), 60-75. [Salov G.I. Power of Nonparametric Tests for Detecting Extended Objects on a Random Background. Avtometriya, 1997, 34(3), 60-75. (in Russian)]

[4] Пяткин В.П., Салов Г.И. Статистический подход к задаче обнаружения некоторых структур на аэрокосмических изображениях . Наукоемкие технологии, 2002, 3(3), 52-58. [Pyatkin V.P., Salov G.I. The statistical approach to the problem of finding some structures on aerospace images. Science Intensive Technologies, 2002, 3(3), 52-58. (in Russian)]

[5] Бучнев А.А., Пяткин В.П., Салов Г.И. Выделение кольцевых структур на космических снимках. Труды IX Международного научного конгресса «ГЕО-Сибирь-2013», Новосибирск, 2013, 3-9 [Buchnev A.A., Pyatkin V.P., Salov G.I. Detection of circular structures in satellite images. Proceedings of the IX International scientific congress "GEO-Siberia 2013”, Novosibirsk, 2013, 3-9 (in Russian)]

[6] Kim P.A., Kalantaev P.A., Pyatkin V.P. Cloud Multiagent System for the Database of Natural Resources. Pattern Recognition and Image Analysis, 2015, 25(2), 220-222.

[7] Салов Г.И. Новый статистический критерий для задач с двумя и тремя выборками, более мощный, чем критерии Вилкоксона и Уитни. Автометрия, 2011, 47(4), 58-70. [Salov G.I. New statistical test for problems with two and three samples, which is more powerful than the Wilcoxon and Whitney tests. Avtometriya, 2011, 47(4), 58-70. (in Russian)] 